\title{
The effects of insulin therapy on mortality in diabetic patients undergoing percutaneous coronary intervention
}

\author{
Shaopeng Xu, Bei Wang, Wennan Liu, Chengcheng Wu, Jinyong Huang \\ Cardiovascular Department, Tianjin Medical University General Hospital, Tianjin, China \\ Contributions: (I) Conception and design: S Xu; (II) Administrative support: B Wang; (III) Provision of study materials or patients: S Xu; (IV) \\ Collection and assembly of data: W Liu, C Wu; (V) Data analysis and interpretation: J Huang; (VI) Manuscript writing: All authors; (VII) Final \\ approval of manuscript: All authors. \\ Correspondence to: Shaopeng Xu. Cardiovascular Department, Tianjin Medical University General Hospital, Tianjin, China. Email: xsplzy@163.com.
}

\begin{abstract}
Background: A growing number of studies have reported insulin therapy to be associated with a higher incidence of major adverse cardiac events in diabetic patients with coronary artery disease. However, the relationship between insulin use and the clinical outcomes of patients with diabetes who undergoing percutaneous coronary intervention (PCI) has not been fully clarified.

Methods: A total of 1,069 consecutive patients with diabetes who underwent PCI were enrolled and divided into 2 groups: oral hypoglycemic agents (OHA) group (709 patients) and insulin therapy group (360 patients). The primary and secondary endpoints of this study were all-cause death and cardiac death, respectively.

Results: At baseline, the maximum creatine kinase-MB (CK-MB), plasma glucose, hemoglobin A1c, highsensitivity C-reactive protein (CRP), and creatinine levels were higher, while the left ventricular ejection fraction (LVEF) was lower, in the insulin therapy group than in the OHA group. After propensity score matching of baseline characteristics, for patients treated with insulin, the odds ratios of death from any cause in hospital, within 1 year of surgery, and within 2 years of surgery were 12.03 (95\% CI: 1.486-97.33, $\mathrm{P}=0.020), 10.33$ (95\% CI: 1.21-88.12, $\mathrm{P}=0.033$ ), and 2.99 (95\% CI: 1.22-7.31, $\mathrm{P}=0.016$ ), respectively, and the odds ratios of cardiac death were 10.33 (95\% CI: 1.21-88.12, $\mathrm{P}=0.033$ ), 6.49 (95\% CI: 1.33-31.59, P=0.021), and 5.27 (95\% CI: $1.45-19.13, \mathrm{P}=0.011$ ), respectively. Generalized estimating equations analysis showed the odds ratios of all-cause death and cardiac death for insulin-treated patients to be 4.77 (95\% CI: 1.76-12.95, $\mathrm{P}=0.002)$ and 5.38 (95\% CI: 1.29-22.96, $\mathrm{P}=0.023)$, respectively.
\end{abstract}

Conclusions: Compared with OHA, insulin therapy significantly increases the risk of in-hospital all-cause and cardiac death in patients with diabetes undergoing PCI, and the risk remains significantly at least 2 years after surgery.

Keywords: Percutaneous coronary intervention (PCI); insulin; oral hypoglycemic agents (OHA); mortality

Submitted Feb 17, 2021. Accepted for publication Aug 16, 2021.

doi: 10.21037/atm-21-1911

View this article at: https://dx.doi.org/10.21037/atm-21-1911

\section{Introduction}

In patients undergoing percutaneous coronary intervention (PCI), the short- and long-term mortality rates among patients with comorbid diabetes mellitus (DM) and coronary artery disease (CAD) remain higher than those among non-diabetic patients, despite intensive care. Maintaining hemoglobin (Hb)A1c $<7.0 \%$ has been shown to improve the microvascular outcomes of patients with diabetes (1). However, the optimal strategy of glucose control has yet to be clarified. Although insulin is fundamental for controlling glucose levels, the debate about whether or not insulin use improves patient outcomes is ceaseless. Despite this controversy, from pharmacological studies to clinical trials 
$(2-4)$, there is increasing evidence to suggest that insulin therapy has harmful effects for patients with diabetes accompanied by CAD. Therefore, in the present study, we sought to explore whether insulin therapy has protective or adverse effects in diabetic patients undergoing PCI. We present the following article in accordance with the STROBE reporting checklist (available at https://dx.doi. org/10.21037/atm-21-1911).

\section{Methods}

\section{Study population}

A consecutive series of 1,069 patients with DM and CAD who underwent PCI at Tianjin Medical University General Hospital China was enrolled from 2002 to 2012. The patients were diagnosed as DM if they had a history of $\mathrm{DM}$ or HbA1c above $6.5 \%$ on admission. CAD, including acute myocardial infarction and stable or unstable angina pectoris, was diagnosed based on angiogram results and clinical manifestations. All of the patients had underwent primary or selective PCI. There were no limitations in terms of stents or lesions. The patients were divided into two groups based on the type of hypoglycemic treatment: the oral hypoglycemic agents (OHA) group and the insulin therapy group. The OHA group included patients treated with sulfonylureas, metformin, $\alpha$-glucosidase inhibitors, thiazolidinedione, and glinides. The insulin therapy group included patients treated with insulin alone or combined with OHAs. The following patients were excluded: patients with severe hepatic or renal dysfunction; patients with cancer, patients treated with a diabetes diet plan only, and patients with hematopathy.

Baseline patient characteristics and in-hospital events were confirmed by hospital chart review. Long-term outcome data were obtained through clinical review or telephone review conducted by the researchers. The primary and secondary endpoints of the study were allcause death and cardiac death, respectively. All patients were followed up for 2 years. Clinical information was collected in hospital, and at lyear and 2 years after surgery.

All procedures performed in this study involving human participants were in accordance with the Declaration of Helsinki (as revised in 2013). This study was approved by the Ethical Committee of Tianjin Medical University General Hospital (No. IRB2021-WZ-036). Individual consent for this retrospective analysis was waived.

\section{Statistical analysis}

Patients' baseline characteristics and clinical outcomes were analyzed using SPSS, version 19.0(SPSS Inc., Chicago, IL, USA). Continuous variables at baseline were analyzed with the independent-samples $t$ test and presented as mean \pm standard deviation. Categorical variables at baseline were analyzed with the chi-square test and presented as numbers (percent frequencies). Propensity score matching was used to adjust the baseline characteristics between the two groups. All outcomes during the patients' hospital stay and at 1 year and 2 years after the procedure were analyzed by logistic regression. Cox regression was performed to calculate survival curves before and after propensity score matching. We used generalized estimating equations to estimate the hazard ratios (HRs) of the insulin therapy group compared with the OHA group. $\mathrm{P}<0.05$ was considered to be statistically significant.

\section{Results}

A total of 1,069 patients were enrolled in the study including 360 in the insulin therapy group and 709 in the OHA group. The mean age was $65.58 \pm 10.92$ and $64.38 \pm 10.50$ of patients treated with insulin and OHAs, respectively $(\mathrm{P}=0.081)$. The baseline clinical and angiographic characteristics of all participants are listed in Tables 1 and 2. At baseline, the maximum creatine kinaseMB (CK-MB), plasma glucose, HbA1C, high-sensitivity $\mathrm{C}$-reactive protein (CRP), and creatinine levels were higher, while the left ventricular ejection fraction (LVEF) was lower, in the insulin therapy group than in the OHA group. The Canadian Cardiovascular Society Angina Class was also higher in the insulin therapy group, as was the proportion of patients with myocardial infarction. There was no difference between the two groups with respect to sex, age, blood pressure, CAD history, prior myocardial infarction, prior percutaneous coronary angioplasty, prior coronary artery bypass graft, hyperlipidemia, low-density lipoprotein, smoking, alcohol consumption, or plasma insulin levels. The angiographic characteristics of the two groups were also comparable (Table 2).

Thirty-seven study participants (3.5\%) died in hospital, of whom 25 were in the insulin therapy group (6.9\%) and 12 were in the OHA group (1.7\%). During the $1^{\text {st }}$ year of follow-up, 30 patients (2.8\%) died including 13 in the insulin therapy group (3.6\%) and 17 in the OHA group 
Table 1 Baseline characteristics

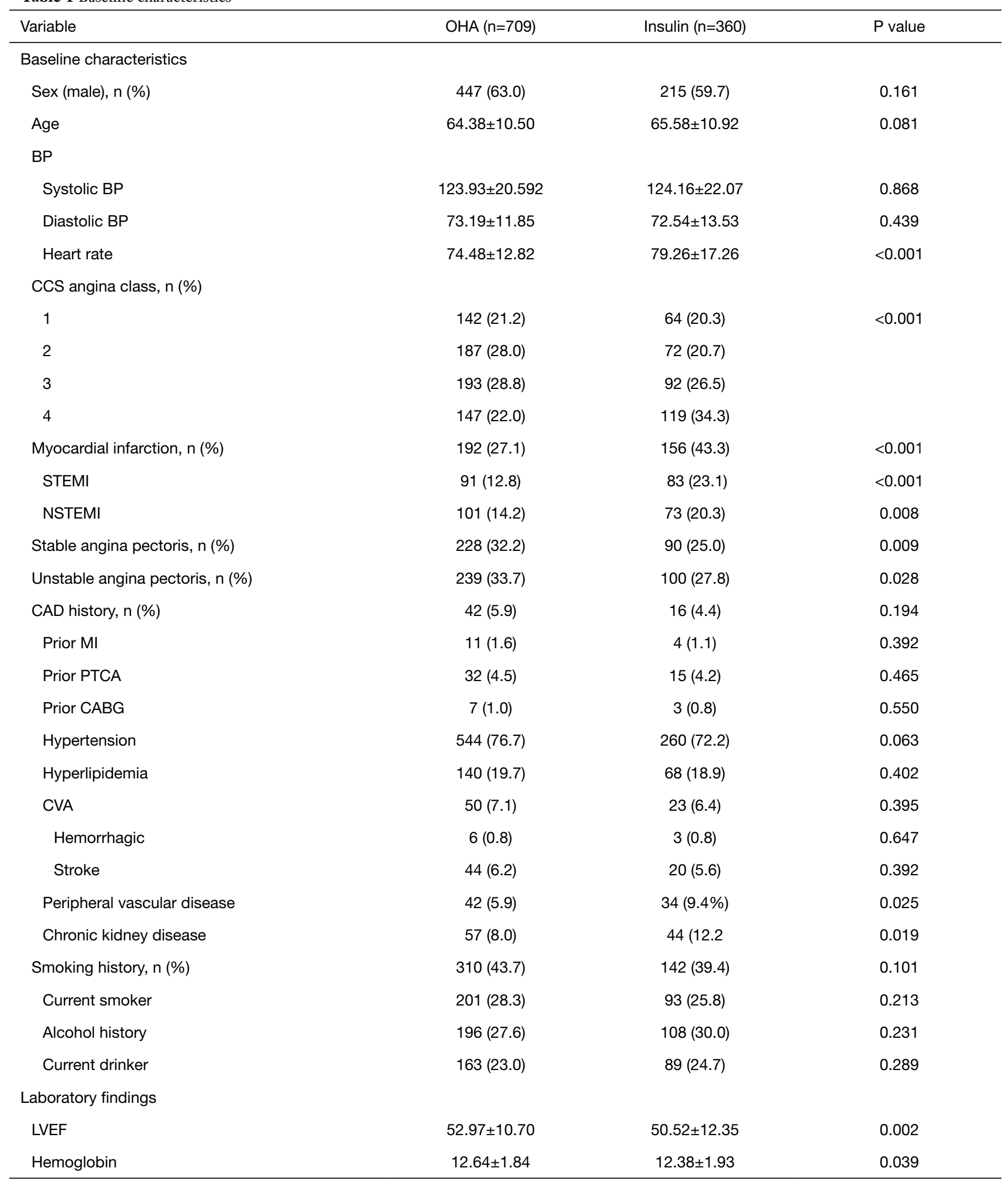

Table 1 (continued) 
Table 1 (continued)

\begin{tabular}{|c|c|c|c|}
\hline Variable & $\mathrm{OHA}(\mathrm{n}=709)$ & Insulin $(n=360)$ & $P$ value \\
\hline CK-MB & $31.96 \pm 81.52$ & $56.66 \pm 118.29$ & 0.001 \\
\hline Troponin T & $0.59 \pm 2.07$ & $0.93 \pm 2.03$ & 0.028 \\
\hline BNP & $1,482.36 \pm 4,828.06$ & $3,284.02 \pm 13,788.31$ & 0.085 \\
\hline Triglyceride & $155.73 \pm 118.33$ & $151.62 \pm 11.78$ & 0.067 \\
\hline HDL cholesterol & $42.61 \pm 10.41$ & $42.74 \pm 11.78$ & 0.882 \\
\hline LDL cholesterol & $103.55 \pm 38.48$ & $105.40 \pm 39.89$ & 0.567 \\
\hline High-sensitivity CRP & $9.09 \pm 23.39$ & $17.23 \pm 39.85$ & 0.001 \\
\hline Insulin & $13.22 \pm 12.80$ & $11.85 \pm 9.82$ & 0.756 \\
\hline C-peptide & $2.98 \pm 1.93$ & $2.81 \pm 2.30$ & 0.399 \\
\hline Creatinine & $1.11 \pm 1.16$ & $1.34 \pm 1.54$ & 0.013 \\
\hline
\end{tabular}

OHA, oral hypoglycemic agents; BP, blood pressure; CCS, Canadian Cardiovascular Society; STEMI, ST-segment Elevation Myocardial Infarction; NSTEMI, non-ST elevation myocardial infarction; CAD, coronary artery disease; MI, myocardial infarction; PTCA, Percutaneous transluminal coronary angioplasty; CABG, coronary artery bypass grafting; CVA, cerebrovascular accident; LVEF, left ventricular ejection fraction; CK-MB, creatine kinase-MB; BNP, B-type natriuretic peptide; HDL, high-density lipoprotein; LDL, low-density lipoprotein; CRP, C-reactive protein.

(2.4\%). In the $2^{\text {nd }}$ year of follow-up, 16 patients $(1.5 \%)$ died including 7 in the insulin therapy group (1.9\%) and 9 in the OHA group (1.3\%). Table 3 details all deaths and cardiac deaths among the study population. Figure 1 and Figure 2 show the rates of all-cause and cardiac death for each year.

The HRs of all-cause and cardiac mortality in hospital, and within 1 year and 2 years after the procedure were analyzed by logistic regression analysis with the covariates of sex, age, acute myocardial infarction, LVEF, plasma glucose levels, HbA1c levels, high-sensitivity CRP levels, and plasma creatinine levels. After regression analysis, the risk factors for death from any cause within 2 years after surgery included age (HR 1.075, 95\% CI: 1.037-1.113, $\mathrm{P}<0.001$ ), creatinine levels (HR 1.286, 95\% CI: 1.060 1.561, $\mathrm{P}=0.011$ ), LVEF (HR 0.947, 95\% CI: 0.926-0.968, $\mathrm{P}<0.001$ ), and high-sensitivity CRP levels (HR 1.011, 95\% CI: $1.005-1.018, \mathrm{P}=0.001)$. The risk factors for cardiac death within 2 years after the procedure included male sex (HR 5.806, 95\% CI: 1.951-17.279, $\mathrm{P}=0.002$ ), age (HR $1.080,95 \%$ CI: $1.033-1.130, \mathrm{P}=0.001)$, insulin therapy (HR 2.474, 95\% CI: 1.050-5.824, P=0.038), LVEF (HR
0.919, 95\% CI: 0.892-0.946, $\mathrm{P}<0.001)$, and high-sensitivity CRP (HR 1.010, 95\% CI: 1.003-1.017, P=0.006). After propensity score matching of glucose levels and HbA1c levels (Tables 4 and 5), the HR of mortality within 2 years after surgery was analyzed again. The risk factors for all-cause mortality included age (HR 1.060, 95\% CI: $1.019-1.103, \mathrm{P}=0.004)$, insulin therapy (HR 2.988, 95\% CI: 1.221-7.310, $\mathrm{P}=0.016$ ), LVEF (HR 0.958, 95\% CI: 0.932 0.984, $\mathrm{P}=0.002$ ), and high-sensitivity CRP (HR 1.010, 95\% CI: $1.003-1.017, \mathrm{P}=0.004)$. We also analyzed the HR of mortality in the insulin therapy group with generalized estimating equation. The HR of all-cause death was 2.428 (95\% CI: $1.238-4.764, \mathrm{P}=0.010)$ and 4.772 (95\% CI: $1.758-12.954, \mathrm{P}=0.002)$ before and after propensity score matching, respectively. The odds ratio of cardiac death was 2.344 (95\% CI: 1.081-5.082, $\mathrm{P}=0.031$ ) and 5.375 (95\% CI: $1.258-22.963, \mathrm{P}=0.023)$ before and after propensity score matching, respectively (Table 6, Figures 3,4). After propensity score matching, insulin therapy was found to be a risk factor for both all-cause and cardiac mortality in hospital, and within 1 year and 2 years of follow-up. The patients' 2-year 
Table 2 Angiographic findings

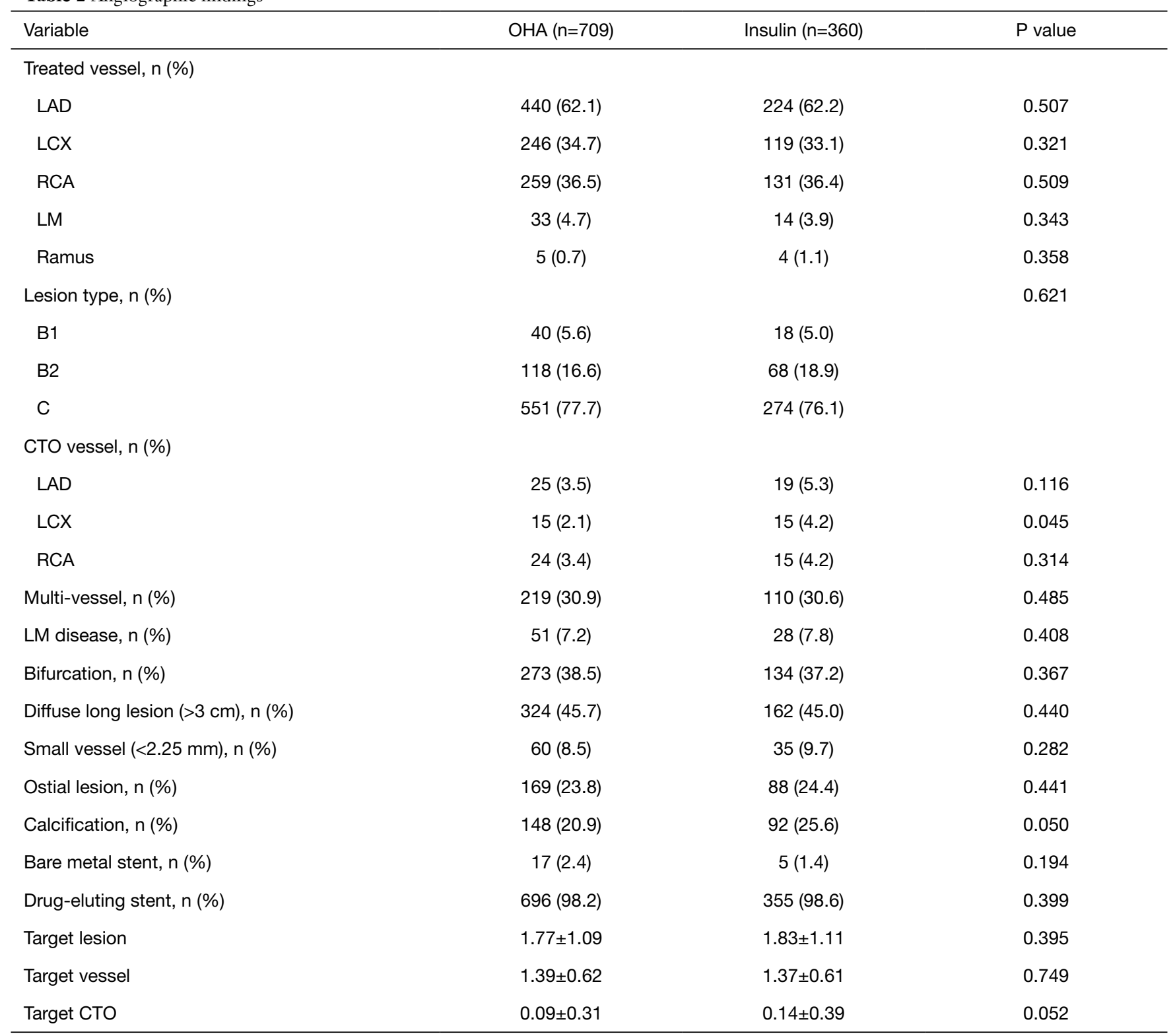

OHA, oral hypoglycemic agents; LAD, left anterior descending artery; LCX, left circumflx branch; RCA, right coronary artery; LM, left main; CTO, chronic total occlusion.

cumulative survival was analyzed by Cox regression before and after propensity score matching; in the insulin therapy group, the HR was 1.917 (95\% CI: 1.044-3.518, $\mathrm{P}=0.036$ ) and 2.89 (95\% CI: $1.218-6.860, \mathrm{P}=0.016)$, respectively (Figures 3 and 5).

After the analysis in all patients enrolled, we analyzed the HR of mortality in patients who were discharged from hospital safely. All-cause mortality and cardiac mortality within 2 years were still higher in insulin-treated patients than in the OHA group; however, the difference between the groups was not statistically significant. In the OHA group and the insulin-treated group, the incidence of allcause death was $3.2 \%$ and $5.6 \%$ (HR 1.60, 95\% CI: $0.563-$ 4.546, $\mathrm{P}=0.378)$, respectively, and that of cardiac death was $1.3 \%$ and $2.7 \%$ (HR 2.782, 95\% CI: $0.540-14.338$, $\mathrm{P}=0.221)$, respectively. 
Table 3 Mortality in the insulin group and OHA group

\begin{tabular}{|c|c|c|c|c|c|c|}
\hline Mortality & \multicolumn{2}{|c|}{ Insulin group, n (\%) } & \multicolumn{2}{|c|}{ OHA group, n (\%) } & \multicolumn{2}{|c|}{ Total, n (\%) } \\
\hline \multicolumn{7}{|c|}{ All-cause mortality } \\
\hline Inhospital & $25(6.9)$ & $25(6.9)$ & $12(1.7)$ & $12(1.7)$ & $37(3.5)$ & $37(3.5)$ \\
\hline Within 1 year & $13(3.6)$ & $38(10.6)$ & $17(2.4)$ & $29(4.1)$ & $30(2.8)$ & $67(6.3)$ \\
\hline \multicolumn{7}{|l|}{ Cardiac mortality } \\
\hline Inhospital & $22(6.1)$ & $22(6.1)$ & $9(1.3)$ & $9(1.3)$ & $31(2.9)$ & $31(2.9)$ \\
\hline Within 1 year & $5(1.4)$ & $27(7.5)$ & $8(1.1)$ & $17(2.4)$ & $13(1.2)$ & $44(4.1)$ \\
\hline Within 2 years & $3(0.8)$ & $30(8.3)$ & $2(0.3)$ & $19(2.7)$ & $5(0.7)$ & $49(4.6)$ \\
\hline
\end{tabular}

OHA, oral hypoglycemic agents.

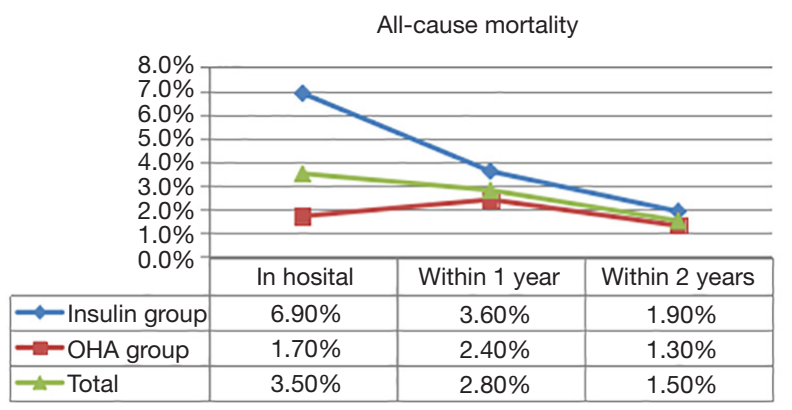

Figure 1 All-cause mortality. The hospital mortality of insulin group was significantly higher than that of oral hypoglycemic drug group. In two years, it gradually became the same.

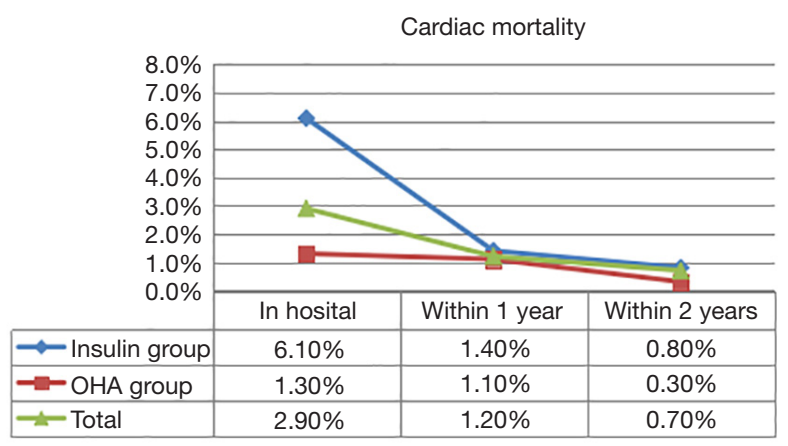

Figure 2 Cardiac mortality. In comparison of cardiovascular mortality between insulin group and oral hypoglycemic drug group, the in-hospital mortality of insulin group was significantly higher than that of oral hypoglycemic drug group. In two years, it gradually became the same.

\section{Discussion}

In a real-world study published recently, in the diabetes patients with CAD, PCI or CABG is associated with lower MACEs, especially the mortality rate, compared with medical treatment. Despite recent advances in medical management and coronary revascularization, cardiovascular disease still accounts for approximately $75 \%$ of all hospital admissions and $80 \%$ of deaths among patients with diabetes (5). Patients with DM undergoing PCI have a higher risk of experiencing major adverse cardiovascular events (MACEs) than non-diabetic patients (6). Elevated plasma glucose levels are well known to be associated with increased patient mortality. However, clinical trials of insulin for intensive glucose control have produced inconsistent results with respect to mortality, which was consistently attributed to suboptimal target glucose levels or the occurrence of hypoglycemia, as shown in the ACCORD (Action to Control Cardiovascular Risk in Diabetes) study (3).

Recently, there has been a trend to scrutinize the effects of oral antidiabetic drugs (OADs) and insulin on diabetes-related complications including micro- and macro-vascular diseases as well as cancer (7). Recently, the safety of insulin in the treatment of type $2 \mathrm{DM}$ (T2DM) has been subject to scrutiny. Among patients with T2DM, it has been reported that exogenous insulin therapy is associated with an increased risk of diabetesrelated complications, cancer, and all-cause mortality. Taking metformin monotherapy as a reference, Currie et al. reported that the adjusted HRs of all-cause 
Table 4 Baseline characteristics of the OHA group and the insulin group after propensity score matching

\begin{tabular}{|c|c|c|c|}
\hline Variable & $\mathrm{OHA}(\mathrm{n}=317)$ & Insulin $(n=317)$ & $P$ value \\
\hline Sex (male), n (\%) & $203(64.0)$ & $188(59.3)$ & 0.253 \\
\hline Age & $63.81 \pm 10.20$ & $65.11 \pm 10.89$ & 0.123 \\
\hline \multicolumn{4}{|l|}{$\mathrm{BP}$} \\
\hline Diastolic BP & $72.86 \pm 11.30$ & $72.64 \pm 13.48$ & 0.823 \\
\hline Heart rate & $74.20 \pm 13.16$ & $78.28 \pm 16.83$ & 0.105 \\
\hline \multicolumn{4}{|l|}{ CCS angina class, $\mathrm{n}(\%)$} \\
\hline 1 & $54(18.3)$ & $56(18.5)$ & 0.139 \\
\hline 4 & $73(24.7)$ & $96(31.5)$ & \\
\hline Myocardial infarction, n (\%) & 99 (31.2) & $131(41.3)$ & 0.010 \\
\hline STEMI & $47(14.9)$ & $70(22.1)$ & 0.024 \\
\hline NSTEMI & $52(16.4)$ & $61(19.2)$ & 0.407 \\
\hline Stable angina pectoris, $\mathrm{n}(\%)$ & $91(28.7)$ & $78(24.6)$ & 0.281 \\
\hline Unstable angina pectoris, $\mathrm{n}(\%)$ & $106(33.4)$ & $94(29.7)$ & 0.347 \\
\hline CAD history, $\mathrm{n}(\%)$ & $17(5.4)$ & $13(4.1)$ & 0.575 \\
\hline Prior Ml & $5(1.6)$ & $3(0.9)$ & 0.725 \\
\hline Hemorrhagic & $4(1.3)$ & $3(0.9)$ & 1.000 \\
\hline Stroke & $18(5.7)$ & $19(6.0)$ & 0.868 \\
\hline Peripheral vascular disease & $13(4.1)$ & $23(7.3)$ & 0.121 \\
\hline Chronic kidney disease & $15(4.7)$ & $33(10.4)$ & 0.010 \\
\hline Smoking history, n (\%) & $145(45.7)$ & 131 (41.3) & 0.298 \\
\hline Current smoker & $93(29.3)$ & $84(26.5)$ & 0.479 \\
\hline Alcohol history & $85(26.8)$ & $98(30.9)$ & 0.293 \\
\hline Current drinker & $70(22.1)$ & $81(25.6)$ & 0.351 \\
\hline \multicolumn{4}{|l|}{ Laboratory findings } \\
\hline LVEF\% & $52.71 \pm 10.72$ & $51.17 \pm 12.07$ & 0.106 \\
\hline Hemoglobin & $12.70 \pm 1.83$ & $12.44 \pm 1.92$ & 0.081 \\
\hline
\end{tabular}

Table 4 (continued) 
Table 4 (continued)

\begin{tabular}{|c|c|c|c|}
\hline Variable & $\mathrm{OHA}(\mathrm{n}=317)$ & Insulin $(n=317)$ & $P$ value \\
\hline CK-MB & $31.74 \pm 90.83$ & $51.43 \pm 115.24$ & 0.086 \\
\hline Troponin T & $0.73 \pm 2.50$ & $0.80 \pm 1.91$ & 0.731 \\
\hline BNP & $1,658.45 \pm 5,517.68$ & $3,335.94 \pm 14,274.83$ & 0.146 \\
\hline Triglyceride & $166.85 \pm 1134.50$ & $155.10 \pm 120.81$ & 0.347 \\
\hline HDL cholesterol & $42.60 \pm 9.79$ & $43.32 \pm 11.80$ & 0.496 \\
\hline LDL cholesterol & $105.94 \pm 37.27$ & $104.75 \pm 39.89$ & 0.759 \\
\hline High-sensitivity CRP & $9.59 \pm 25.45$ & $15.92 \pm 36.95$ & 0.018 \\
\hline Insulin & $11.26 \pm 7.04$ & $12.97 \pm 10.18$ & 0.373 \\
\hline C-peptide & $3.00 \pm 2.01$ & $2.80 \pm 2.30$ & 0.386 \\
\hline Creatinine & $1.09 \pm 1.21$ & $1.31 \pm 1.53$ & 0.046 \\
\hline
\end{tabular}

OHA, oral hypoglycemic agents; BP, blood pressure; CCS, Canadian Cardiovascular Society; STEMI, ST-segment Elevation Myocardial Infarction; NSTEMI, non-ST elevation myocardial infarction; CAD, coronary artery disease; MI, myocardial infarction; PTCA, percutaneous transluminal coronary angioplasty; CABG, coronary artery bypass grafting; CVA, cerebrovascular accident; LVEF, left ventricular ejection fraction; CK-MB, creatine kinase-MB; BNP, B-type natriuretic peptide; HDL, high-density lipoprotein; LDL, low-density lipoprotein; CRP, C-reactive protein.

mortality, myocardial infarction, and MACEs for insulin therapy were $2.197,1.954$, and 1.736 , respectively (8). Another recently published study conducted by Roumie et al. demonstrated that the addition of insulin was associated with an increased risk of nonfatal cardiovascular outcomes and all-cause mortality when compared to sulfonylurea among patients with diabetes who were receiving metformin (9). Also, the adjusted $\mathrm{HR}$ of all-cause mortality for insulin therapy was $1.44(\mathrm{P}=0.001 ; 33.7$ and 22.7 per 1000 person-years) compared with OHA. A study of 56,536 patients with T2DM showed pioglitazone to be associated with significantly reduced all-cause mortality (HR 0.33) compared to insulin (10). Based on an average followup of 20 years, Kurl concluded that insulin resistance and insulin, but not fasting plasma glucose, are independent risk factors for death caused by coronary heart disease (11).

In an observational cohort study conducted by Younk and colleagues in more than 12,000 newly treated individuals with T2DM, the risk of all-cause mortality, cardiovascular mortality, and non-cardiovascular mortality increased in patients with insulin exposure (2). Another observational cohort study conducted by Gamble in 2010 also showed a graded risk of all-cause mortality, cardiovascular mortality, and non-cardiovascular mortality to be associated with increasing levels of insulin exposure, and the adjusted HR for high insulin exposure was $2.79(\mathrm{P}=0.005)$ (12).

Among patients undergoing PCI, Abizaid et al.'s 1998 study demonstrated that patients with insulin-treated DM had a higher risk of in-hospital mortality and a significantly lower cardiac event-free survival rate. In the same study, inhospital mortality among insulin-treated patients were $2 \%$, which was significantly higher than the rate among OHAtreated patients (4). A similar result was found in the BARI trial (13). In patients undergoing coronary artery bypass grafting, cardiac survival at 5 and 10 years was related to insulin treatment status but not to diabetes(14). Patients with insulin-treated DM had lower survival than both non-DM patients and patients with non-insulin-treated $\mathrm{DM}(\mathrm{P}<0.0001)$; however, cardiac survival was comparable between non-diabetic and diabetic patients receiving insulin therapy.

In patients with acute myocardial infarction undergoing 
Table 5 Baseline angiographic findings after propensity score matching

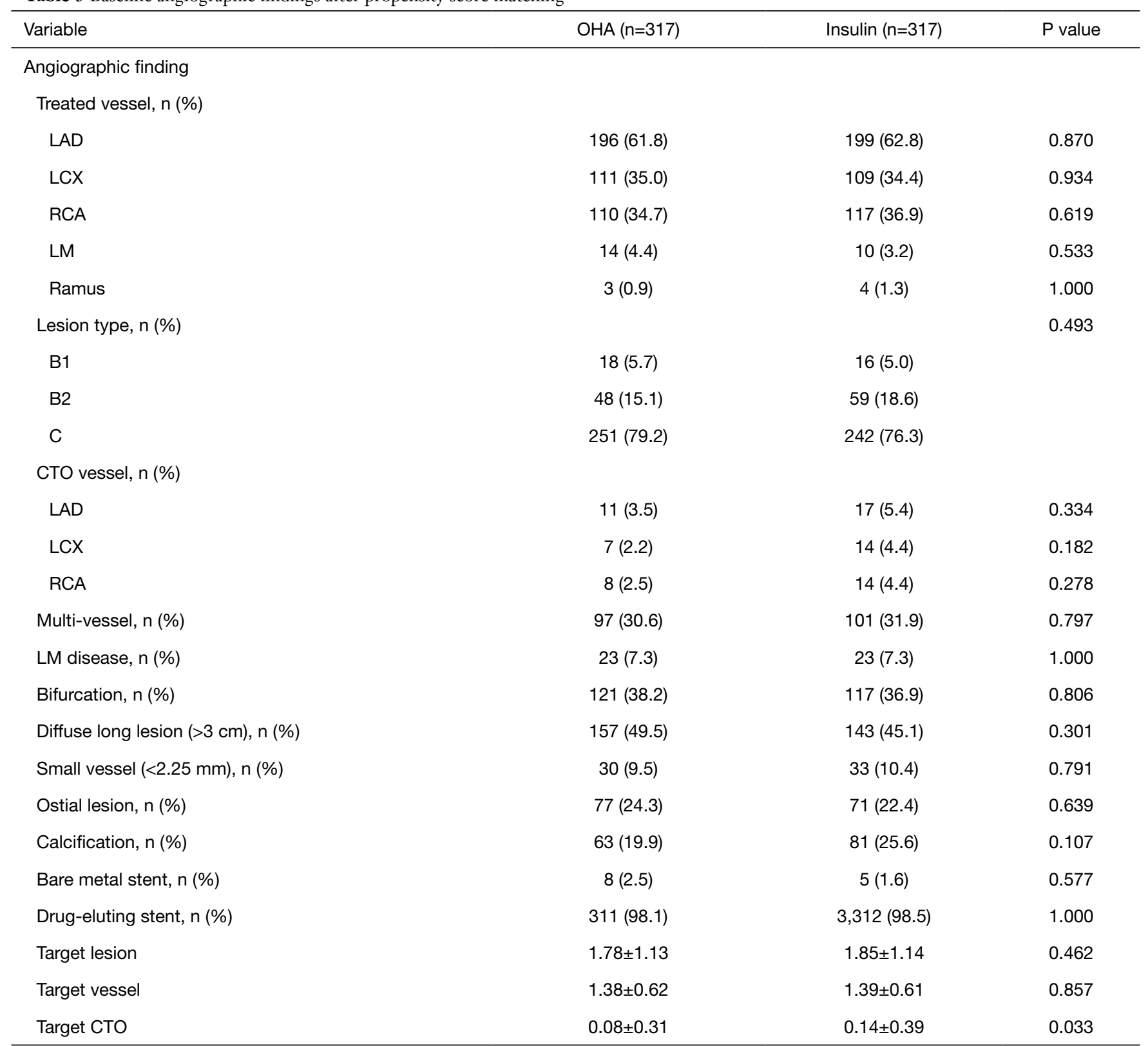

OHA, oral hypoglycemic agents; LAD, left anterior descending artery; LCX, left circumflx branch; RCA, right coronary artery; LM, left main; CTO, chronic total occlusion.

Table 6 Risk factors for mortality in hospital and within 1 and 2 years

\begin{tabular}{|c|c|c|c|c|c|c|}
\hline Risk factors & \multicolumn{3}{|c|}{ Before matching } & \multicolumn{3}{|c|}{ After matching } \\
\hline \multicolumn{7}{|l|}{ Inhospital } \\
\hline \multicolumn{7}{|l|}{ All-cause mortality } \\
\hline Insulin therapy & 4.269 & $1.443-12.628$ & 0.009 & 12.025 & $1.486-97.329$ & 0.020 \\
\hline
\end{tabular}

Table 6 (continued) 
Table 6 (continued)

\begin{tabular}{|c|c|c|c|c|c|c|}
\hline Risk factors & \multicolumn{3}{|c|}{ Before matching } & \multicolumn{3}{|c|}{ After matching } \\
\hline LVEF & 0.940 & $0.909-0.972$ & $<0.001$ & 0.944 & $0.907-0.983$ & 0.005 \\
\hline HsCRP & 1.012 & $1.004-1.020$ & 0.003 & 1.014 & $1.005-1.022$ & 0.003 \\
\hline \multicolumn{7}{|l|}{ Cardiac mortality } \\
\hline HsCRP & 1.013 & $1.004-1.021$ & 0.003 & 1.015 & $1.006-1.024$ & 0.002 \\
\hline \multicolumn{7}{|l|}{ Within 1 year } \\
\hline \multicolumn{7}{|l|}{ All-cause mortality } \\
\hline Insulin therapy & 3.148 & $1.459-6.788$ & 0.003 & 10.109 & $2.255-45.320$ & 0.003 \\
\hline Male & 4.194 & $1.688-10.424$ & 0.002 & 3.609 & $1.242-10.484$ & 0.018 \\
\hline Age & 1.092 & $1.047-1.138$ & $<0.001$ & 1.082 & $1.030-1.137$ & 0.002 \\
\hline \multicolumn{7}{|l|}{ Cardiac mortality } \\
\hline Insulin therapy & 2.726 & $1.059-7.019$ & 0.038 & 6.486 & $1.332-31.590$ & 0.021 \\
\hline LVEF & 0.909 & $0.879-0.939$ & $<0.001$ & 0.930 & $0.894-0.968$ & $<0.001$ \\
\hline HsCRP & 1.009 & $1.002-1.017$ & 0.014 & 1.012 & $1.004-1.020$ & 0.004 \\
\hline Male & 10.118 & $2.757-37.136$ & $<0.001$ & 5.569 & $1.409-22.005$ & 0.014 \\
\hline Age & 1.095 & $1.041-1.152$ & $<0.001$ & 1.083 & $1.022-1.148$ & 0.007 \\
\hline Male & 2.065 & $0.972-4.386$ & 0.059 & 1.485 & $0.629-3.508$ & 0.367 \\
\hline Age & 1.081 & $1.041-1.123$ & $<0.001$ & 1.060 & $1.019-1.103$ & 0.004 \\
\hline \multicolumn{7}{|l|}{ Cardiac mortality } \\
\hline Insulin therapy & 2.474 & $1.050-5.824$ & 0.038 & 5.271 & $1.453-19.131$ & 0.011 \\
\hline LVEF & 0.919 & $0.892-0.946$ & $<0.001$ & 0.939 & $0.908-0.971$ & $<0.001$ \\
\hline HsCRP & 1.010 & $1.003-1.017$ & 0.006 & 1.012 & $1.004-1.020$ & 0.004 \\
\hline Male & 5.806 & $1.951-17.279$ & 0.002 & 1.485 & $0.629-3.508$ & 0.367 \\
\hline Age & 1.008 & $1.033-1.130$ & 0.001 & 1.069 & $1.023-1.118$ & 0.003 \\
\hline \multicolumn{7}{|l|}{ Overall } \\
\hline \multicolumn{7}{|l|}{ All-cause mortality } \\
\hline Insulin therapy & 2.428 & $1.238-4.764$ & 0.010 & 4.772 & $1.758-12.954$ & 0.002 \\
\hline \multicolumn{7}{|l|}{ Cardiac mortality } \\
\hline Insulin therapy & 2.344 & $1.081-5.082$ & 0.031 & 5.375 & 1.258-22.963 & 0.023 \\
\hline
\end{tabular}

HR, hazard ratios; LVEF, left ventricular ejection fraction; HsCRP, high sensitive $\mathrm{C}$ reacting protein. 


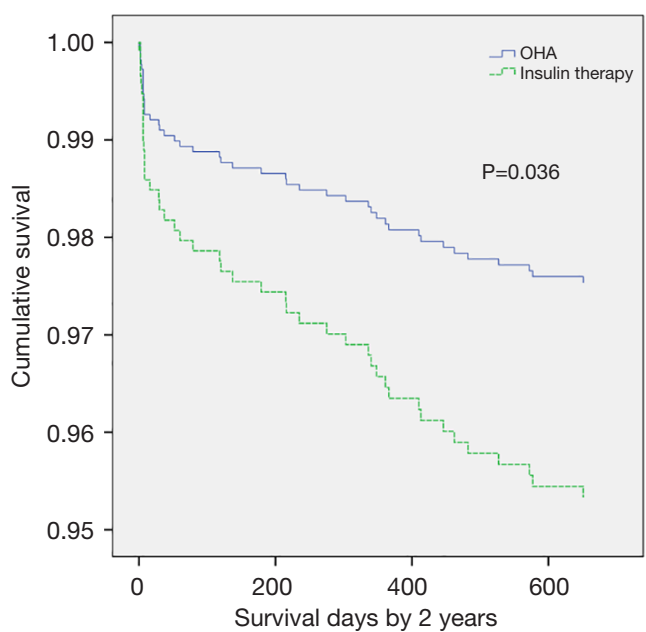

Figure 3 Two-year cumulative survival before propensity score matching. Before PSM matching, the survival rate of insulin group was significantly lower than that of oral hypoglycemic drug group.

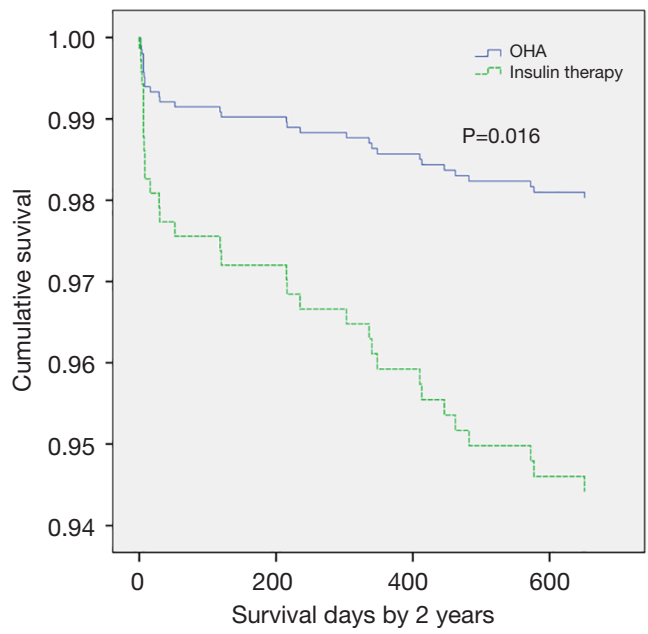

Figure 4 Two-year cumulative survival after propensity score matching. After PSM matching, the survival rate of insulin group was significantly lower than that of oral hypoglycemic drug group.

primary PCI, insulin-treated diabetes is a predictor of 6-month mortality. In Antoniucci et al.'s study, the mortality rate was $26 \%$ in the insulin-treated DM group, which was significantly higher than the rates of $7 \%$ and $4 \%$ in the non-DM group and non-insulin-treated DM group, respectively. Most deaths occurred during hospitalization. The authors speculated that the excess mortality among patients with DM who required insulin may be explained not only by their poor baseline profiles but also by the lack

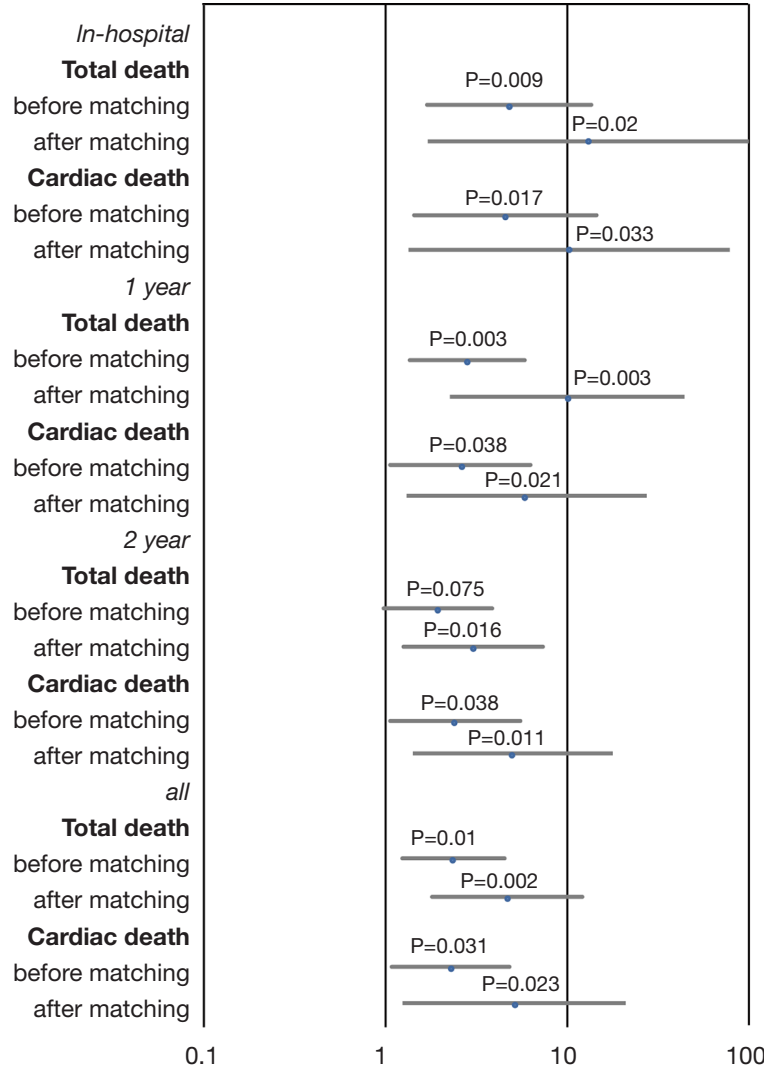

Figure 5 Forest plot of odds ratios of mortality in the insulin therapy group. The mortality of insulin group and oral hypoglycemic drug group was significantly higher than that of oral hypoglycemic drug group.

of myocardial reperfusion observed in many insulin-treated patients, as evidenced by a low incidence of early STsegment resolution (15).

However, previous studies have arrived at conflicting conclusions. For instance, in 2004, Mathew et al. reported that insulin treatment was not independently associated with poor survival in diabetic patients who successfully underwent PCI. However, in that study, patients in the control group were treated with a diabetes diet and/or OHAs. The glucose levels in the OHA group were not as high as those in Insulin group (16). The groups differed in terms of baseline characteristics; in the insulin group, there were more females and more patients with complications, a history coronary bypass or coronary angioplasty, a history of myocardial infarction, or congestive heart failure, which may have blurred the results.

António et al. reported insulin treatment to be an 
independent predictor of neither 1-year mortality nor MACEs. Diabetic patients receiving insulin had similar outcomes to those not receiving insulin despite having more advanced atherosclerotic disease (17). However, baseline characteristics were not balanced between the groups, with the insulin group having a higher prevalence of prior myocardial infarction and stroke or transient ischemic attack, as well as higher glucose levels, than the non-insulin group; such imbalance at baseline decreases the value of study findings.

Similarly, the ORIGIN study failed to demonstrate a significant increase in macrovascular events with the use of insulin glargine (18). There was also no difference in the incidence of cancer or cancer mortality between patients treated with and without insulin. However, patients in the insulin group had more weight gain and suffered more hypoglycemic events.

In the BARI-2D study, the authors drew the opposite conclusion to the BARI study, finding no significant difference in mortality or MACEs between strategies of insulin sensitization and insulin provision (19). However, some patients in the insulin-sensitization group were given insulin injection to reach the target HbA1c level.

The findings of the DIGAMI 2 study strongly support the concept of meticulous glucose control, rather than insulin treatment or the insulin dose, being the factor that improves cardiac outcomes in patients with hyperglycemia. DIGAMI-2 also found that insulin treatment was associated with an increased risk of nonfatal cardiovascular events (OR $1.89, \mathrm{P}=0.0002)$, regardless the non-significant difference in all-cause mortality and cardiovascular events (2).

In some of the above-mentioned studies which compared intensive treatment and standard treatment and produced non-significant results with respect to mortality, differences inpatient baseline characteristics may, at least in part, explain the poor outcomes seen in insulin-treated patients (4,15-17). Furthermore, some studies were designed to explore the association of glucose targets with mortality and cardiac outcomes of patients, rather than the favorable or adverse effects of insulin on mortality (19). Thus, these studies randomized participants according to glucose levels but not treatment strategy, making it difficult to analyze the effects of insulin on outcomes. For instance, in the ACCORD trial, insulin was used to treat $68 \%$ and $58 \%$ of participants in the intensive treatment and standard treatment groups, respectively; in the ADVANCE trial, these proportions were $41 \%$ and $24 \%$, respectively. Therefore, we cannot speculate on the effects of insulin on patient outcomes based on those studies.

Since the question of whether the lower target glucose level or insulin therapy is the main mechanism responsible for the adverse effects of intensive glucose control has not yet been answered, we designed the present study to address it in diabetic patients undergoing PCI. The findings of this study were similar to those of the study conducted by Abizaid et al. However, in Abizaid et al.'s study, all patients enrolled were treated with bare metal stents (4), whereas in the present study, the overwhelming majority of patients enrolled were stented with drug eluting stents. Moreover, the baseline glucose levels differed between the two groups in Abizaid et al.'s study, whereas we use propensity score matching to balance our patients' baseline characteristics, especially the levels of glucose and HbA1c. In the present study. after matching, insulin-treated patients still exhibited significantly higher all-cause mortality and cardiac mortality than non-insulin-treated patients, suggesting that longterm insulin treatment is associated with an elevated risk of mortality in hospital and during follow-up in diabetic patients undergoing PCI. Among patients who were safely discharged, the rates of all-cause death and cardiac death showed no difference between the OHA and insulin groups, which suggested that the difference in mortality observed in hospital persisted for at least 2 years after surgery.

\section{Mechanism}

The concentration of proinsulin, which is a surrogate marker of IR and $\beta$-cell stress, has been found to be an independent predictor of all-cause mortality in diabetic patients $(20,21)$. Hyperinsulinemia has been significantly associated with cardiovascular mortality in both males and females, independent of other risk factors (22). Among patients with diabetes, studies have indicated that elevated insulin levels predict adverse outcomes $(4,23)$ and that controlling hyperglycemia by reducing insulin resistance, rather than by providing insulin, might improve cardiovascular outcomes.

Some reports have shown that insulin exerts antiinflammatory and anti-atherogenic effects and improves endothelial function; in contrast, other studies have suggested that insulin therapy is associated with increased vascular resistance, ventricular hypertrophy, and endothelial dysfunction (17). The cardiovascular outcomes of these trials were discordant, possibly because the benefit of glucose reduction in patients with diabetes can be counterbalanced by the atherogenic effect of hyperinsulinemia in patients 
treated with intensive glucose control (11).

Here, we hypothesize that short-term use of insulin in the acute stage of acute coronary syndrome may attenuate inflammation and oxidative stress, but long-term use of insulin may diminish the sensitivity of tissue to insulin or increase insulin resistance. This is why, in DIGAMI study, low mortality was especially evident in patients who had a low cardiovascular risk profile and no previous insulin treatment. For patients with diabetes, the long-term use of insulin worsens outcomes, as the favorable effects evoked by insulin, such as anti-apoptotic and anti-inflammatory effects, eventually fade (24). It is well known that insulin causes more weight gain than sulphonylurea (1). Whether or not insulin treatment attenuates the insulin sensitivity of cells has yet to be clarified; however, long-term insulin use can potentially aggravate insulin resistance through its adverse effects of fluid retention and obesity. Similar phenomena can be observed in drug resistance in other treatments.

The authors of studies which suggested that intensive glucose control has adverse effects have consistently attributed these effects to hypoglycemia in insulin-treated patients. However, a direct link between hypoglycemia and cardiovascular complications in this population of patients with multimorbidities has not been evidenced (25). Based on the present study and previous research, hypoglycemia is not the main or sole reason for the increased mortality risk in patients receiving insulin.

\section{Limitations}

The present study has a number of limitations. First, this study is retrospective in nature. During follow-up, neither the levels of glucose or HbA1c, nor patients' insulin therapy status were recorded, nor were data on insulin concentration available. Also, the duration of insulin treatment, which may be a basic characteristic influencing prognosis, was not available for analysis. Furthermore, data on the occurrence of hypoglycemia during follow-up were not recorded. However, the mean HbAlc in the two groups was approximately $8 \%$, which suggested that the occurrence of hypoglycemia was low.

Secondly, different OHAs influence outcomes differently. Monotherapy with the most commonly used insulin secretagogues appears to be associated with increased mortality and cardiovascular risk compared with metformin (26). In the present study, we did not record the types of OHA, so we were unable to differentiate insulin secretagogues and sensitizers. Since insulin secretagogues also increase plasma insulin levels, secretagogues and sensitizers may have different effects with regard to improving insulin resistance and mortality, and this might have influenced the results of the present study.

\section{Conclusions}

Insulin therapy may be inferior to OHA therapy as a longterm treatment for diabetes. Insulin use significantly increases the risk of in-hospital all-cause mortality and cardiac mortality in patients undergoing PCI compared with OHA therapy, and the risk remains significantly for at least 2 years after surgery.

\section{Acknowledgments}

Funding: None.

\section{Footnote}

Reporting Checklist: The authors have completed the STROBE reporting checklist. Available at https://dx.doi. org/10.21037/atm-21-1911

Data Sharing Statement: Available at https://dx.doi, org/10.21037/atm-21-1911

Conflicts of Interest: All authors have completed the ICMJE uniform disclosure form (available at https://dx.doi. org/10.21037/atm-21-1911). The authors have no conflicts of interest to declare.

Ethical Statement: The authors are accountable for all aspects of the work in ensuring that questions related to the accuracy or integrity of any part of the work are appropriately investigated and resolved. All procedures performed in this study involving human participants were in accordance with the Declaration of Helsinki (as revised in 2013). This study was approved by the Ethical Committee of Tianjin Medical University General Hospital (No. IRB2021-WZ-036). Individual consent for this retrospective analysis was waived.

Open Access Statement: This is an Open Access article distributed in accordance with the Creative Commons Attribution-NonCommercial-NoDerivs 4.0 International License (CC BY-NC-ND 4.0), which permits the non- 
commercial replication and distribution of the article with the strict proviso that no changes or edits are made and the original work is properly cited (including links to both the formal publication through the relevant DOI and the license). See: https://creativecommons.org/licenses/by-nc-nd/4.0/.

\section{References}

1. Intensive blood-glucose control with sulphonylureas or insulin compared with conventional treatment and risk of complications in patients with type 2 diabetes (UKPDS 33). UK Prospective Diabetes Study (UKPDS) Group. Lancet 1998;352:837-53.

2. Younk LM, Lamos EM, Davis SN. The cardiovascular effects of insulin. Expert Opin Drug Saf 2014;13:955-66

3. Gerstein HC, Miller ME, Byington RP, et al. Effects of intensive glucose lowering in type 2 diabetes. $\mathrm{N} \mathrm{Engl} \mathrm{J}$ Med 2008;358:2545-59.

4. Abizaid A, Kornowski R, Mintz GS, et al. The influence of diabetes mellitus on acute and late clinical outcomes following coronary stent implantation. J Am Coll Cardiol 1998;32:584-9.

5. Kassaian SE, Goodarzynejad H, Boroumand MA, et al. Glycosylated hemoglobin (HbA1c) levels and clinical outcomes in diabetic patients following coronary artery stenting. Cardiovasc Diabetol 2012;11:82.

6. Sharma PK, Agarwal S, Ellis SG, et al. Association of Glycemic Control With Mortality in Patients With Diabetes Mellitus Undergoing Percutaneous Coronary Intervention. Circ Cardiovasc Interv 2014;7:503-9.

7. Weng J, Yang X. Unsubstantiated concerns over the safety of use of sulphonylureas and insulin for increased risk of diabetes complications. J Diabetes 2014;6:30-2.

8. Currie CJ, Poole CD, Evans M, et al. Mortality and other important diabetes-related outcomes with insulin vs other antihyperglycemic therapies in type 2 diabetes. J Clin Endocrinol Metab 2013;98:668-77.

9. Roumie CL, Greevy RA, Grijalva CG, et al. Association between intensification of metformin treatment with insulin vs sulfonylureas and cardiovascular events and all-cause mortality among patients with diabetes. JAMA 2014;311:2288-96.

10. Yang J, Vallarino C, Bron M, et al. A comparison of allcause mortality with pioglitazone and insulin in type 2 diabetes: an expanded analysis from a retrospective cohort study. Curr Med Res Opin 2014;30:2223-31.

11. Kurl S, Zaccardi F, Onaemo VN, et al. Association between HOMA-IR, fasting insulin and fasting glucose with coronary heart disease mortality in nondiabetic men: a 20year observational study. Acta Diabetol 2015;52:183-6.

12. Gamble JM, Simpson SH, Eurich DT, et al. Insulin use and increased risk of mortality in type 2 diabetes: a cohort study. Diabetes Obes Metab 2010;12:47-53.

13. Influence of diabetes on 5-year mortality and morbidity in a randomized trial comparing CABG and PTCA in patients with multivessel disease: the Bypass Angioplasty Revascularization Investigation (BARI). Circulation 1997;96:1761-9.

14. Mohammadi S, Dagenais F, Mathieu P, et al. Longterm impact of diabetes and its comorbidities in patients undergoing isolated primary coronary artery bypass graft surgery. Circulation 2007;116:1220-5.

15. Antoniucci D, Valenti R, Migliorini A, et al. Impact of insulin-requiring diabetes mellitus on effectiveness of reperfusion and outcome of patients undergoing primary percutaneous coronary intervention for acute myocardial infarction. Am J Cardiol 2004;93:1170-2.

16. Mathew V, Frye RL, Lennon R, et al. Comparison of survival after successful percutaneous coronary intervention of patients with diabetes mellitus receiving insulin versus those receiving only diet and/or oral hypoglycemic agents. Am J Cardiol 2004;93:399-403.

17. António N, Soares F, Lourenço C, et al. Impact of previous insulin therapy on the prognosis of diabetic patients with acute coronary syndromes. Arq Bras Endocrinol Metabol 2010;54:612-9.

18. ORIGIN Trial Investigators; Gerstein HC, Bosch J, et al. Basal insulin and cardiovascular and other outcomes in dysglycemia. N Engl J Med 2012;367:319-28.

19. BARI 2D Study Group; Frye RL, August P, et al. A randomized trial of therapies for type 2 diabetes and coronary artery disease. N Engl J Med 2009;360:2503-15.

20. Alssema M, Dekker JM, Nijpels G, et al. Proinsulin concentration is an independent predictor of all-cause and cardiovascular mortality: an 11-year follow-up of the Hoorn Study. Diabetes Care 2005;28:860-5.

21. Farhan S, Jarai R, Tentzeris I, et al. Admission proinsulin is associated with mortality in patients with admission hyperglycemia during acute coronary syndrome: results from a pilot observational study. Clin Chem 2011;57:1456-60.

22. DECODE Insulin Study Group. Plasma insulin and cardiovascular mortality in non-diabetic European men and women: a meta-analysis of data from eleven prospective studies. Diabetologia 2004;47:1245-56.

23. Kronmal RA, Barzilay JI, Tracy RP, et al. The relationship 
of fasting serum radioimmune insulin levels to incident coronary heart disease in an insulin-treated diabetic cohort. J Clin Endocrinol Metab 2004;89:2852-8.

24. Marfella R, Di Filippo C, Portoghese M, et al. Tight glycemic control reduces heart inflammation and remodeling during acute myocardial infarction in hyperglycemic patients. J Am Coll Cardiol 2009;53:1425-36.

Cite this article as: $\mathrm{Xu} \mathrm{S}$, Wang $\mathrm{B}$, Liu W, Wu C, Huang J. The effects of insulin therapy on mortality in diabetic patients undergoing percutaneous coronary intervention. Ann Transl Med 2021;9(16):1294. doi: 10.21037/atm-21-1911
25. Hanefeld M, Bramlage P. Insulin use early in the course of type 2 diabetes mellitus: the ORIGIN trial. Curr Diab Rep 2013;13:342-9.

26. Schramm TK, Gislason GH, Vaag A, et al. Mortality and cardiovascular risk associated with different insulin secretagogues compared with metformin in type 2 diabetes, with or without a previous myocardial infarction: a nationwide study. Eur Heart J 2011;32:1900-8. 